\title{
LONGITUDINAL TUNE-UP OF SNS NORMAL CONDUCTING LINAC *
}

\author{
Dong-o Jeon ${ }^{\dagger}$, SNS/ORNL, P.O.Box 2008, Oak Ridge, TN37830, USA \\ J. Stovall, R. Shafer, LANL, Los Alamos, NM87545, USA \\ K. Crandall, TechSource, Santa Fe, NM87594, USA
}

\begin{abstract}
DTL of SNS linac accelerates the $2.5 \mathrm{MeV} \mathrm{H}^{-}$beam from RFQ and MEBT to $86 \mathrm{MeV}$. For longitudinal setpoint, two standard phase scan methods will be used, because they are complementary. Numerical simulation using Parmila code indicates that only the phase scan with the absorber and collector is effective for DTL tank 6. But for the rest DTL tanks, both methods are effective.
\end{abstract}

\section{INTRODUCTION}

Commissioning of DTL comprises longitudinal setpoint ( $\mathrm{rf}$ amplitude and phase setting), transverse matching from the Medium Energy Beam Transport (MEBT) and closed orbit correction using dipole correctors. In this paper, two widely used phase scan methods are studied for the longitudinal set-point. One is phase scan with two downstream BPMs (Beam Position Monitor) and the other phase scan with the absorber and collector (foil and Faraday Cup). Simulations are performed to see if these methods can be applied to SNS normal conducting linac using the PARMILA code [1].

\section{PHASE SCAN WITH TWO DOWNSTREAM BPMS}

Using two down-stream BPMs, beam bunch phase can be measured. Comparing simulation and measurements, rf amplitude and phase can be set. Schematic plot of this scheme is in Fig. 1. The two down-stream BPMs of DTL tank 1 is inside DTL tank 2. They are $6 \beta \lambda$ apart (a complete period).

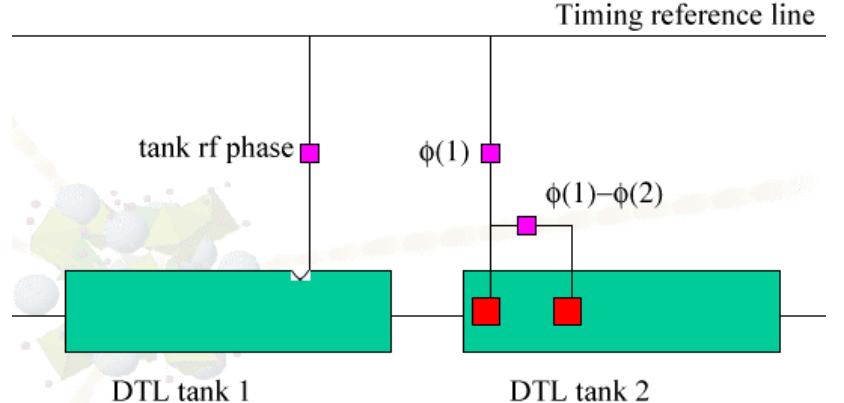

BPM (Beam Position Monitor) BPMs are apart by $6 \beta \lambda$ (one period)

Figure 1: Schematic plot of phase scan with two downstream BPMs.

*Work supported by the DOE, under contract No. DE-AC0500OR22725 with UT-Batelle, LLC for ORNL

†jeond@ornl.gov
Phase advance plays an important role in this technique and is a function of tank rf amplitude and the offset from the design rf phase. As an example, particle trajectories are plotted for two different rf amplitude of DTL tank 1 in Fig. 2. Pink loci are trajectories of beam bunches with different injection phase at the end of DTL tank 1.
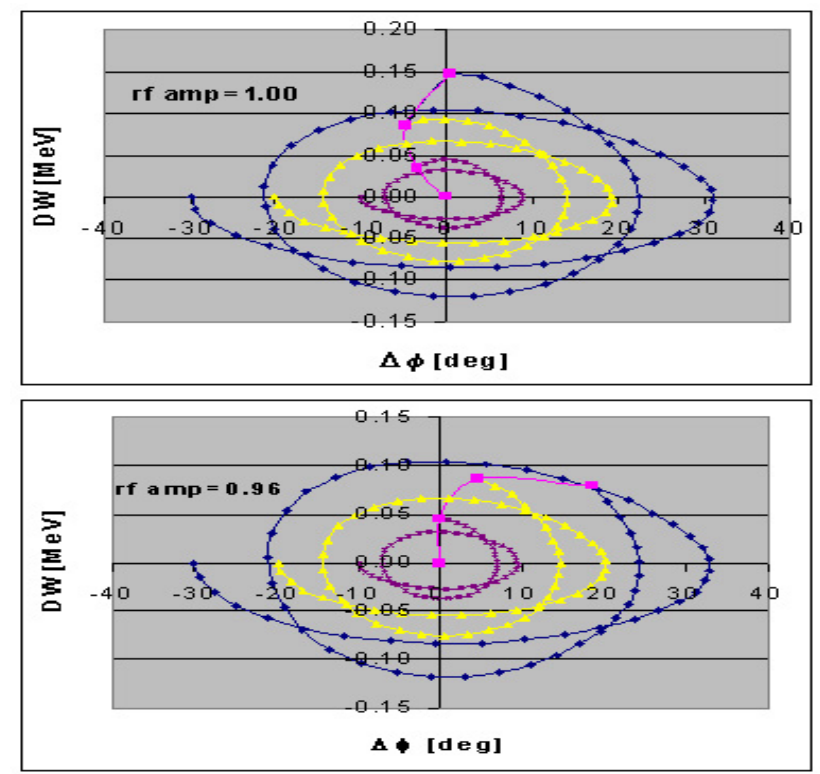

Figure 2: Phase advance plots for two different $r f$ amplitudes: 1.00 of design value (top) and 0.96 (bottom).

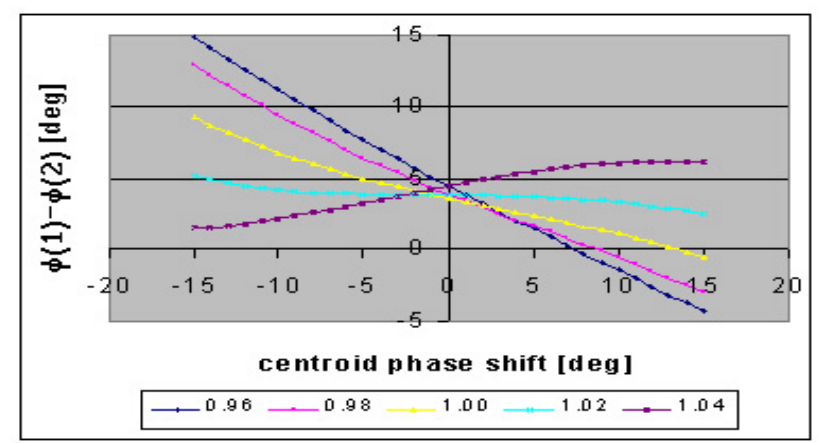

Figure 3: phase difference between two BPM signals vs. centroid phase shift for five different tank rf amplitudes. This plot is for DTL tank 1.

In Fig. 3, $\mathrm{x}$-axis is the deviation from the design bunch phase and $y$-axis is for the phase difference between two detected BPM signals. Different curves stand for different tank rf amplitude. For the rf amplitude of 1.02 (meaning $102 \%$ of design rf amplitude), phase difference becomes almost independent of bunch injection phase shift from the design. From this, the rf amplitude can be determined. After rf amplitude is determined, rf phase can be easily 
determined from the crossing point of two different $\mathrm{rf}$ amplitudes. The same plot can be used for DTL tank 2 and 5 in a similar manner.

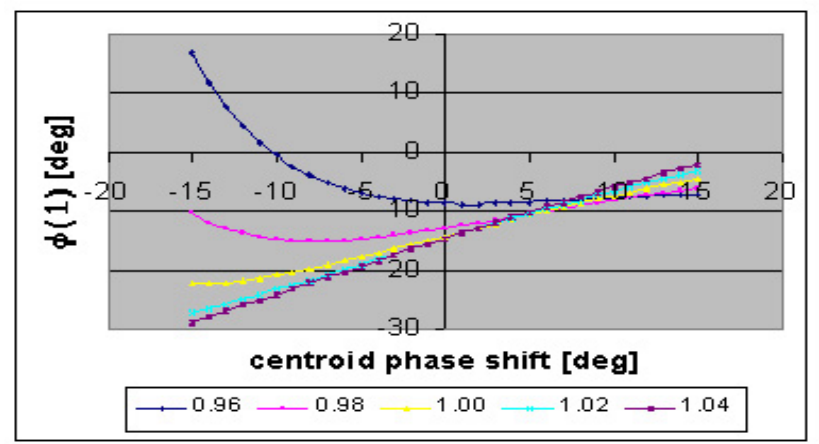

Figure 4: Beam phase of BPM 1 signal for five different tank rf amplitudes. This is for DTL tank 3.

In the case of DTL tank 3 and 4, phase from the BPM 1 turns out useful rather than the phase difference. Similarly, there is an rf amplitude where part of the BPM phase becomes independent of bunch injection phase shift. By comparing measurement with simulation, the tank rf amplitude can be determined. After rf amplitude is determined, $\mathrm{rf}$ phase can be easily determined from the crossing point of two different rf amplitudes.

In the case of DTL tank 6, neither the phase difference nor the phase of one BPM is effective as is shown in Fig. 5. So an alternative method should be used, called phase scan with the absorber and collector.
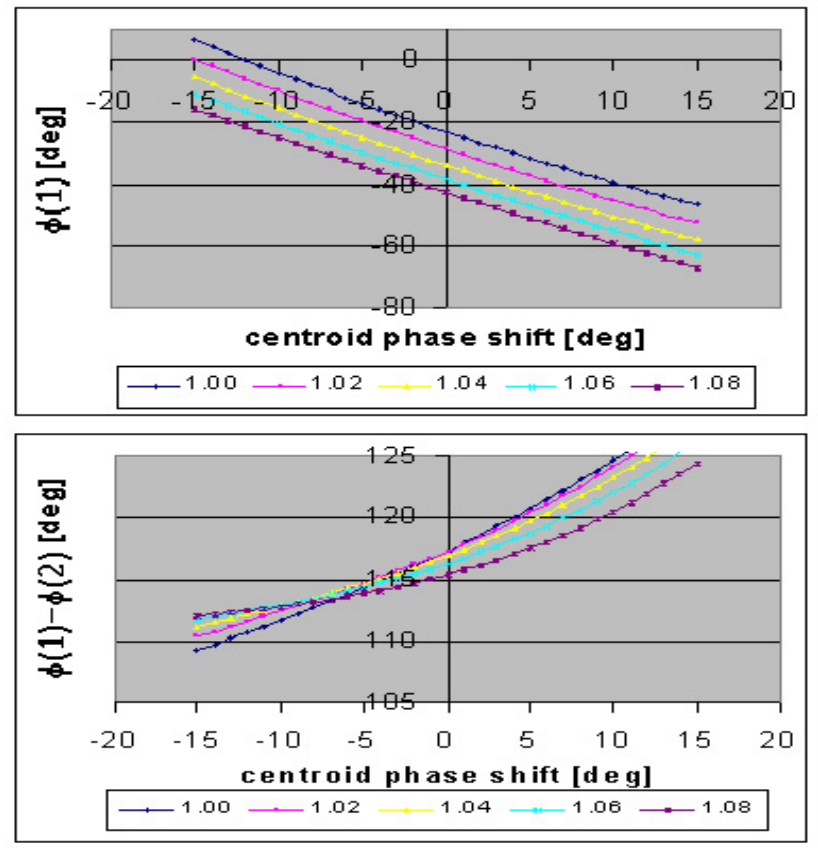

Figure 5: BPM phase plots vs. tank rf amplitude (top) and BPM phase difference plots vs. tank rf amplitude (bottom). This is for DTL tank 6.

\section{PHASE SCAN WITH THE ABSORBER AND COLLECTOR}

Another widely used method for longitudinal set-point is the phase scan with the absorber (foil) and collector (Faraday Cup). The absorber removes low energy tail of beam bunch and the surviving beam is collected using the Faraday Cup. A schematic plot of this scheme is shown in Fig. 6.

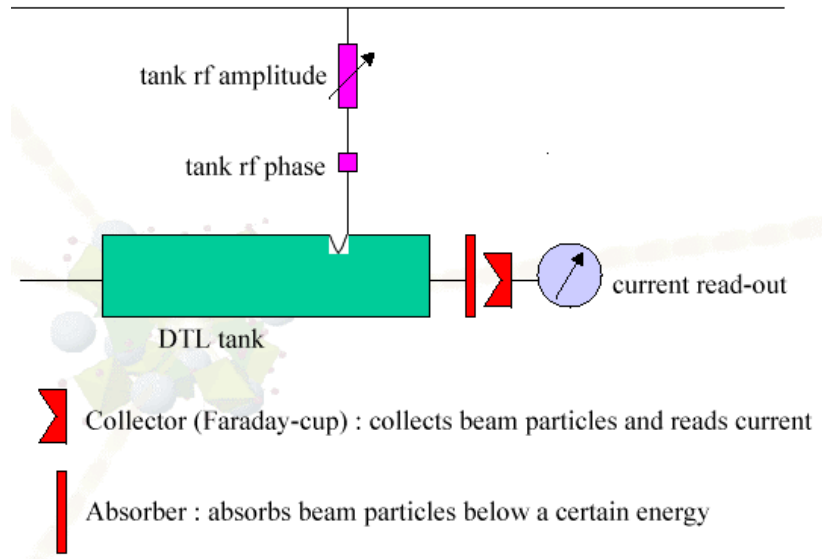

Figure 6: Schematic drawing of phase scan with the absorber and collector.

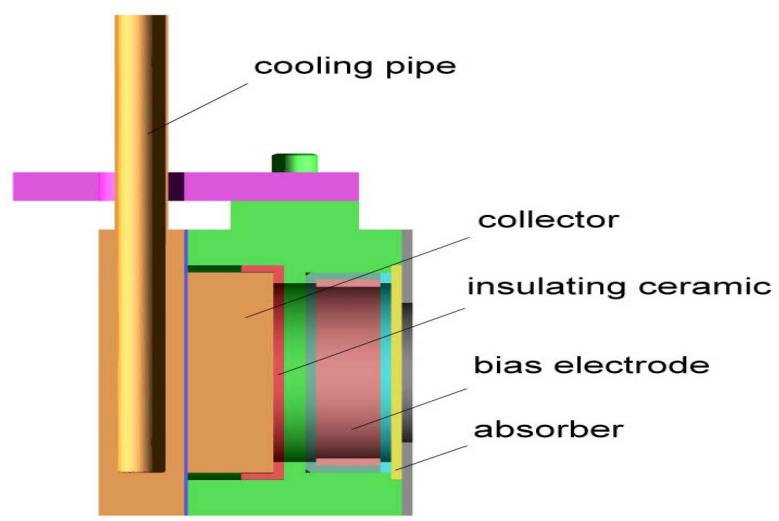

Figure 7: Plot of the absorber and collector assembly. Beam is incident from the right.

Figure 7 is the plot of the absorber and collector assembly. The absorber and collector is an in-line device mounted on actuators. The collector can take up to $50 \mu \mathrm{s}$ full current beam pulse at $1 \mathrm{~Hz}$ at $185 \mathrm{MeV}$, which corresponds to $300 \mathrm{~W}$ maximum.

The success of this method depends heavily on the proper choice of the threshold energy of an absorber. In order to determine the threshold energy, the acceptance plot and the energy spectrum plot of the beam are utilized. In Fig. 8, acceptance plots of DTL tank 3 are shown for three different rf amplitudes, $0.9,1.0$, and 1.1. This shows that tail has energy below $38 \mathrm{MeV}$. Figure 9 shows the energy spectrum of beam vs. beam centroid phase offset $\Delta \phi$ from the design with the nominal tank rf amplitude. From Figs. 8 and 9, threshold energy of the absorber can be chosen to be $38 \mathrm{MeV}$. 


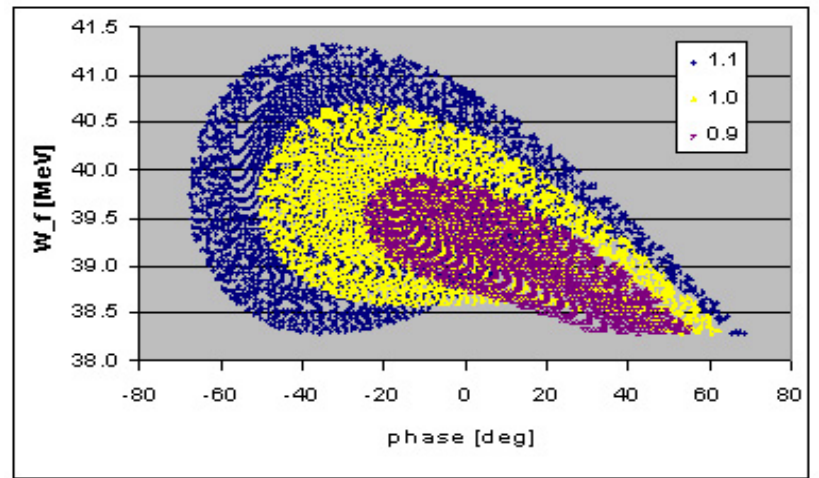

Figure 8: Acceptance plot at the output of DTL tank 3.

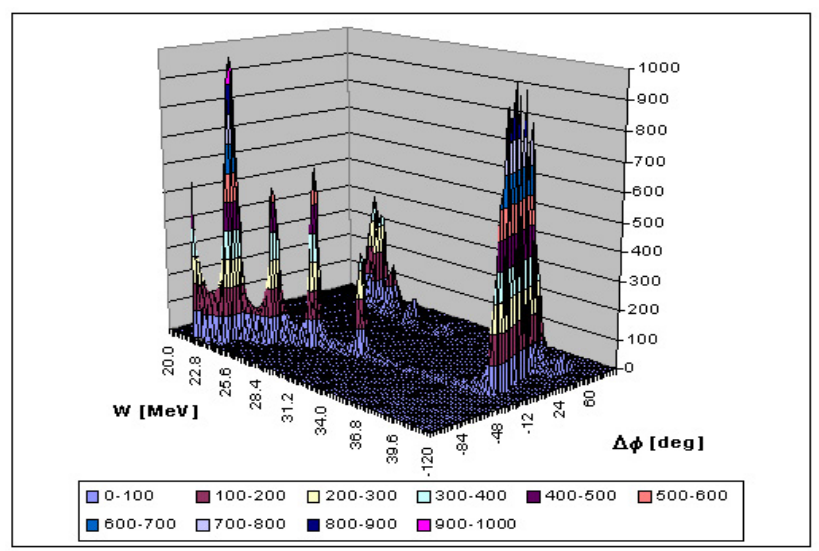

Figure 9: Energy spectrum of beam vs. beam centroid offset $\Delta \phi$ from the design. Z-axis is proportional to current.

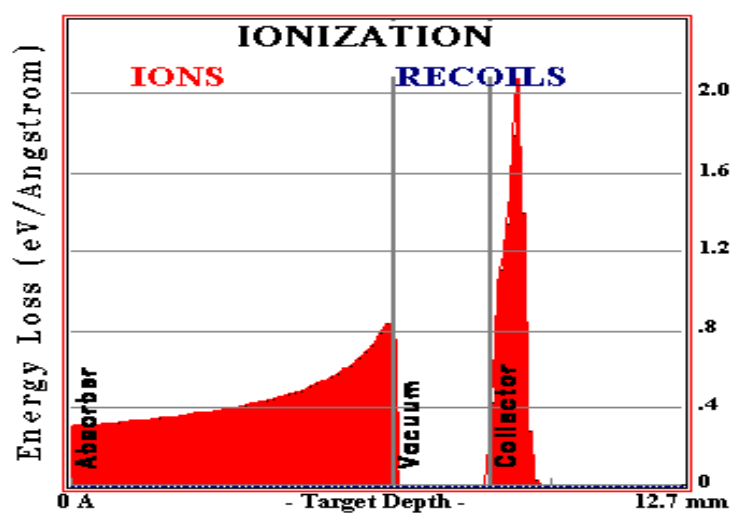

Figure 10: Trim simulation of $38 \mathrm{MeV}$ absorber and collector. Nominal beam energy is $39.8 \mathrm{MeV}$ from DTL tank 3. Most of beam stops in the collector.

Figure 10 shows the Trim simulation of $39.8 \mathrm{MeV}$ beam out of DTL tank 3 through a $38 \mathrm{MeV}$ Carbon absorber $(6.72 \mathrm{~mm}$ thickness) and a collector. As is expected, most of beam stops in the collector.

Now multiparticle simulations are done with an absorber and collector assembly at the end of a DTL tank. Top plot of Fig. 11 shows the normalized current read from the collector after DTL tank 3. By comparing the Full-Width-Half-Maximum width of experiment and simulation in Fig. 11, tank rf amplitude can be determined. The red dot is the design value. Also from the points of half maximum, phase can be determined.

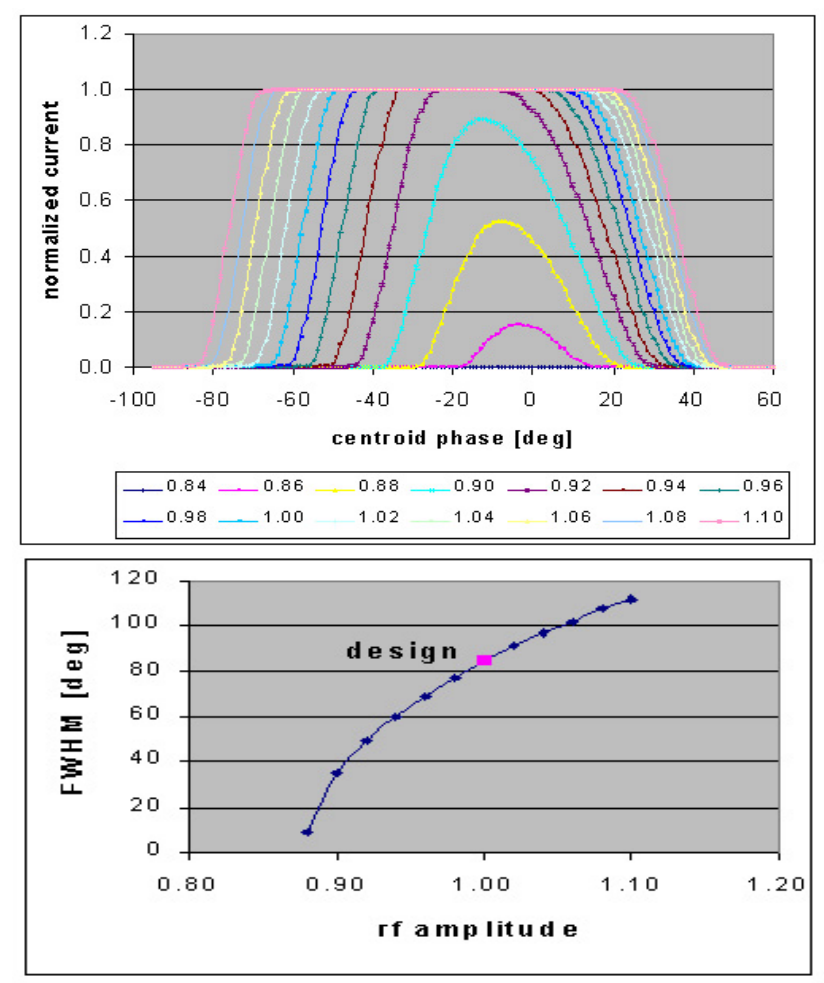

Figure 11: Normalized beam current read from the collector vs. tank rf amplitude (top) and the corresponding Full-Width-Half-Maximum width of DTL tank 3.

\section{SUMMARY}

Through numerical simulations using the PARMILA code, it has been demonstrated that DTL rf amplitude and phase can be set using the two standard phase scan methods.

\section{REFERENCES}

[1] H. Takeda and J. Stovall, "Modified PARMILA code for new accelerating structures", Proceedings of the 1995 Particle Accelerator Conference, p.2364 (Dallas, Texas). 